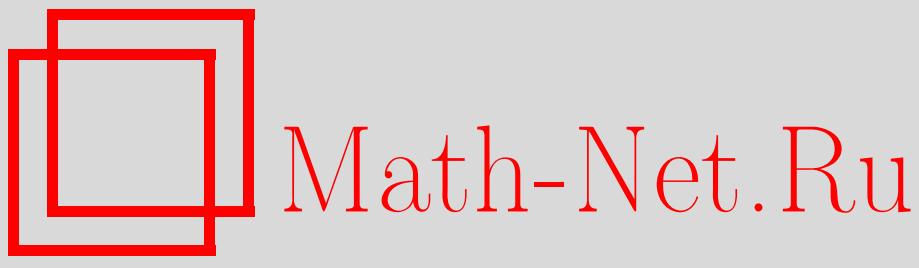

М. А. Ольшанецкий, В. К. Рогов, Модифицированные $q$-функции Бесселя и $q$ функции Макдональда, Матем. сб., 1996, том 187, номер 10, 109-128

DOI: https://doi.org/10.4213/sm167

Использование Общероссийского математического портала Math-Net.Ru подразумевает, что вы прочитали и согласны с пользовательским соглашением

http://www . mathnet.ru/rus/agreement

Параметры загрузки:

IP: 52.87 .193 .239

26 апреля 2023 г., $17: 24: 12$ 
УДК 517.58

\author{
М. А. Ольшанецкий, В.-Б.К. Рогов
}

\title{
Модифицированные $q$-функции Бесселя и $q$-функции Макдональда
}

\begin{abstract}
В работе определяются $q$-аналоги модифицированных функций Бесселя и функции Макдональда. Как и $q$-функции Бесселя, введенные Джексоном, эти функции бывают двух видов. Подобно их классическим прототипам они возникают в гармоническом анализе на квантовых симметрических пространствах. Их определение основано на представлении в виде степенного ряда. Получены рекуррентные соотношения, разностные уравнения, $q$-вронскианы и аналоги асимптотических разложений, которые являются сходящимися рядами, если $q \neq 1$. Даются некоторые следующие отсюда соотношения для базисных гипергеометрических рядов.

Библиография: 13 названий.
\end{abstract}

\section{§1. Введение}

В последние годы вновь стали предметом исследования $q$-аналоги функций Бесселя [2], [3], введенные девяносто лет тому назад Джексоном в [1]. В указанных работах свойства $q$-функций Бесселя получены в связи с теорией представлений квантовых групп так же, как и в классическом случае [4].

Для нас главный интерес представляют собой $q$-аналоги функции Макдональда $K_{\nu}(\Phi \mathrm{M})$ и модифицированной функции Бесселя $I_{\nu}(\mathrm{MФБ).} \mathrm{Их} \mathrm{присутствие}$ в гармоническом анализе на однородных пространствах обусловлено, по крайней мере, тремя факторами. Прежде всего, ФМ является существенной частью функции Грина для оператора Лапласа-Бельтрами на некомпактных симметрических пространствах неположительной кривизны [5], [6]. Далее, ФМ определяют неприводимые представления группы изометрий псевдоевклидовой плоскости [4]. Наконец, ФМ являются простейшими, так называемыми, функциями Уитеккера [7]-[9], связанными с симметрическими пространствами ранга 1. Оказывается, функции Уитеккера калибровочного преобразования совпадают с волновыми функциями открытой квантовой цепочки Тоды [9]. В частности, для симметрических пространств ранга 1 они определяют волновые функции двумерной гравитации [10].

Основная мотивация нашей работы заключается в следующем. В предыдушей работе [11] мы описали функции Уитеккера на квантовом пространстве Лобачевского. Они возникают как собственные функции оператора Казимира второго порядка некоторого специального вида. В такой постановке задача, которая изначально является некоммутативной, может быть сведена к задаче коммутативного

Работа первого автора выполнена при поддержке РФФИ (грант № 94-02-14365), МНФ (грант № MIF-300) и ИНТАС (грант № 93-0166).

Работа второго автора выполнена при поддержке МНФ (грант № JC 7100). 
анализа. Дальнейшее продвижение в гармоническом анализе требует принимать во внимание некоммутативность групповой алгебры. Это, в частности, относится к ядру Пуассона задачи Дирихле на квантовом пространстве Лобачевского. В классическом случае функция Уитеккера или, что то же самое, ФМ является преобразованием Фурье ядра Пуассона. Для того чтобы перенести эту конструкцию на квантовый случай, необходимо получить основные свойства $q$-МФБ.

Так же, как и в классическом случае, мы начинаем с определения $q$-МФБ как степенного ряда. Имеются две $q$-МФБ и они связаны с $q$-функциями Бесселя Джексона [1] так же, как и в классическом случае. Мы получаем для них действия разностных операторов, рекуррентные соотношения, разностные уравнения и $q$-вронскианы. Большинство этих результатов может быть легко получено из [2], [3], где исследовались $q$-функции Бесселя. В то время как определение $q$-МФБ основано непосредственно на джексоновских $q$-функциях Бесселя, определение $q$-ФМ более тонко. Мы даем такое определение $q-\Phi М$, чтобы она была голоморфной в правой полуплоскости. Для $q-\Phi М$ мы повторяем ту же программу, что и для $q-$ МФБ. Наиболее важными являются разложения лорановского типа, представляющие собой ряды, сходящиеся в некоторой области, аналоги которых в классике являются только асимптотическими. Используя полученные свойства, мы даем в заключение некоторые соотношения для базисных гипергеометрических рядов.

\section{§ 2. Некоторые предварительные сведения}

Приведем некоторые необходимые соотношения для функций $e_{q}(z), E_{q}(z)$, и $\Gamma_{q}(z)[12]$. Кроме того, определим $q$-пси-функцию.

2.1. Для любого $a$

$$
\begin{gathered}
(a, q)_{n}= \begin{cases}1 & \text { при } n=0 ; \\
(1-a)(1-a q) \cdots\left(1-a q^{n-1}\right) & \text { при } n \geqslant 1 ;\end{cases} \\
(a, q)_{\infty}=\lim _{n \rightarrow \infty}(a, q)_{n} .
\end{gathered}
$$

q-экспоненты определяются формулами

$$
\begin{aligned}
& e_{q}(z)=\sum_{n=0}^{\infty} \frac{z^{n}}{(q, q)_{n}}=\frac{1}{(z, q)_{\infty}}, \quad|z|<1 \\
& E_{q}(z)=\sum_{n=0}^{\infty} \frac{q^{n(n-1) / 2} z^{n}}{(q, q)_{n}}=(-z, q)_{\infty} .
\end{aligned}
$$

Они представляют собой $q$-дефформации обычной экспоненты

$$
\lim _{q \rightarrow 1-0} e_{q}((1-q) z)=\lim _{q \rightarrow 1-0} E_{q}((1-q) z)=e^{z}
$$

Очевидно, из (1) и (2) имеем

$$
e_{q}(z) E_{q}(-z)=1
$$

Из (1) следует, что функция $e_{q}(z)$ имеет простые полюсы в точках $z=q^{-k}$, $k=0,1, \ldots$. 
ПРЕДЛОЖЕНИЕ 2.1 (Ф.И. Карпелевич). q-экспонента $e_{q}(z)$ можст бъть представлена в виде суммъ простейших дробей

$$
e_{q}(z)=\frac{1}{(q, q)_{\infty}} \sum_{k=0}^{\infty} \frac{(-1)^{k} q^{k(k+1) / 2}}{(q, q)_{k}\left(1-z q^{k}\right)}
$$

ДОКАЗАТЕЛЬСТво. Пусть

$$
e_{q}(z, n)=\frac{1}{(q, q)_{n}}=\sum_{k=0}^{n} \frac{c_{k, n}}{1-z q^{k}} .
$$

Тогда

$$
\begin{aligned}
c_{k, n} & =\operatorname{res}_{z=q^{-k}} e_{q}(z, n)=\lim _{z \rightarrow q^{-k}}\left(1-z q^{k}\right) e_{q}(z, n) \\
& =\frac{1}{\left(1-q^{-k}\right) \cdots\left(1-q^{-1}\right)(1-q) \cdots\left(1-q^{n-k}\right)} \\
& =\frac{(-1)^{k} q^{k(k+1) / 2}}{(q, q)_{k}(q, q)_{n-k}} .
\end{aligned}
$$

Отсюда

$$
e_{q}(z, n)=\sum_{k=0}^{n} \frac{(-1)^{k} q^{k(k+1) / 2}}{(q, q)_{k}\left(1-z q^{k}\right)} \frac{1}{(q, q)_{n-k}} .
$$

Так как $1 /(q, q)_{n-k}<1 /(q, q)_{\infty}$, получим

$$
e_{q}(z)=\lim _{n \rightarrow \infty} e_{q}(z, n)=\frac{1}{(q, q)_{\infty}} \sum_{k=0}^{\infty} \frac{(-1)^{k} q^{k(k+1) / 2}}{(q, q)_{k}\left(1-z q^{k}\right)}
$$

Следующее предложение вытекает из (1).

ПРЕДЛОЖЕНИЕ 2.2. Для функиии $e_{q}$ выполнено соотношение

$$
\partial_{q} e_{q}((1-q) z)=e_{q}((1-q) z)
$$

$2 \partial e$

$$
\partial_{q} f(z)=\frac{f(z)-f(q z)}{(1-q) z}
$$

Очевидно следующее

СлЕДСтВИЕ 2.1. Справедливы равенства

$$
\begin{aligned}
e_{q}\left(\frac{1-q^{2}}{2} q z\right) & =\left(1-\frac{1-q^{2}}{2} z\right) e_{q}\left(\frac{1-q^{2}}{2} z\right) \\
e_{q}\left(\frac{1-q^{2}}{2} q^{-1} z\right) & =\frac{1}{1-q^{-1}\left(1-q^{2}\right) z / 2} e_{q}\left(\frac{1-q^{2}}{2} z\right) . \\
e_{q^{2}}\left(\frac{\left(1-q^{2}\right)^{2}}{4} q^{2} z^{2}\right) & =\left(1-\frac{\left(1-q^{2}\right)^{2}}{4} z^{2}\right) e_{q^{2}}\left(\frac{\left(1-q^{2}\right)^{2}}{4} z^{2}\right) .
\end{aligned}
$$


ЗАМЕЧАНИЕ 2.1. Из (1) следует, что

$$
\begin{aligned}
e_{q}\left(\frac{1-q^{2}}{2} z\right) e_{q}\left(-\frac{1-q^{2}}{2} z\right) & =\frac{1}{\left(\frac{1-q^{2}}{2} z, q\right)_{\infty}} \frac{1}{\left(\frac{1-q^{2}}{2} z, q\right)_{\infty}} \\
& =\frac{1}{\left(\frac{\left(1-q^{2}\right)^{2}}{4} z^{2}, q^{2}\right)_{\infty}}=e_{q}^{2}\left(\frac{\left(1-q^{2}\right)^{2}}{4} z^{2}\right) .
\end{aligned}
$$

2.2. $q$-гамма-функция определяется формулой

$$
\Gamma_{q}(\alpha)=\frac{(q, q)_{\infty}}{\left(q^{\alpha}, q\right)_{\infty}}(1-q)^{1-\alpha}
$$

Если $n$ является натуральным числом, то

$$
\Gamma_{q}(n+1)=\frac{(q, q)_{n}}{(1-q)^{n}} .
$$

ПРЕДЛОЖЕНИЕ 2.3. Функиия $\Gamma_{q^{2}}(z)$ может бъть представлена в виде

$$
\Gamma_{q^{2}}(z)=\left(1-q^{2}\right)^{1-z} \sum_{k=0}^{\infty} \frac{(-1)^{k} q^{k(k+1)}}{\left(q^{2}, q^{2}\right)_{k}\left(1-q^{2 k+2 z}\right)} .
$$

ДокаЗАТЕЛЬСТво. Из (9) следует, что

$$
\Gamma_{q^{2}}(z)=\left(q^{2}, q^{2}\right)_{\infty}\left(1-q^{2}\right)^{1-z} e_{q^{2}}\left(q^{2 z}\right) .
$$

Подставляя сюда (4), получим (10).

Обозначим через $\psi_{q}(z)$ логарифмическую производную $\Gamma_{q}$-функции

$$
\psi_{q}(z)=\frac{\Gamma_{q}^{\prime}(z)}{\Gamma_{q}(z)}
$$

Из (9) и (11) сразу же получается

ПРЕДЛОЖЕНИЕ 2.4. Функиия $\psi_{q^{2}}(z)$ имеет следующий вид:

$$
\psi_{q^{2}}(z)=-\ln \left(1-q^{2}\right)+\ln q^{2} \sum_{k=1}^{\infty} \frac{q^{2 k+2 z}}{1-q^{2 k+2 z}} .
$$

СлеДСтвиЕ 2.2. Справедлива формула

$$
\lim _{z \rightarrow-n} \frac{\psi_{q^{2}}(z)}{\Gamma_{q^{2}}(z)}=(-1)^{n} \frac{q^{-n(n+1)}\left(q^{2}, q^{2}\right)_{n}}{\left(1-q^{2}\right)^{n+1}} \ln q^{2} .
$$

ДокАЗАТЕЛЬСтво. Этот результат получается из [10] и (12). 


\section{§ 3. Модифицированные $q$-функции Бесселя}

3.1. Определения. В [1] q-функции Бесселя были определены следующим образом

$$
\begin{aligned}
& J_{\nu}^{(1)}(z, q)=\frac{\left(q^{\nu+1}, q\right)_{\infty}}{(q, q)_{\infty}}\left(\frac{z}{2}\right)^{\nu}{ }_{2} \Phi_{1}\left(0,0 ; q^{\nu+1} ; q,-\frac{z^{2}}{4}\right) \\
& J_{\nu}^{(2)}(z, q)=\frac{\left(q^{\nu+1}, q\right)_{\infty}}{(q, q)_{\infty}}\left(\frac{z}{2}\right)^{\nu}{ }_{0} \Phi_{1}\left(-; q^{\nu+1} ; q,-\frac{z^{2} q^{\nu+1}}{4}\right)
\end{aligned}
$$

где ${ }_{2} \Phi_{1}$ и $0 \Phi_{1}-$ базисные гипергеометрические функции [12],

$$
\begin{aligned}
& { }_{r} \Phi_{s}\left(a_{1}, \ldots, a_{r} ; b_{1}, \ldots, b_{s} ; q, z\right) \\
& \quad=\sum_{n=0}^{\infty} \frac{\left(a_{1}, q\right)_{n} \cdots\left(a_{r}, q\right)_{n}}{(q, q)_{n}\left(b_{1}, q\right)_{n} \cdots\left(b_{s}, q\right)_{n}}\left((-1)^{n} q^{n(n-1) / 2}\right)^{1+s-r} z^{n} .
\end{aligned}
$$

Это позволяет определить модифицированные $q$-функции Бесселя ( $q$-МФБ), исходя из (14) и (15) по аналогии с классическим случаем [13].

ОПРЕДЕЛЕНИЕ 3.1. ФункциИ

$$
\begin{aligned}
& I_{\nu}^{(1)}(z, q)=\frac{\left(q^{\nu+1}, q\right)_{\infty}}{(q, q)_{\infty}}\left(\frac{z}{2}\right)^{\nu}{ }_{2} \Phi_{1}\left(0,0 ; q^{\nu+1} ; q, \frac{z^{2}}{4}\right) \\
& I_{\nu}^{(2)}(z, q)=\frac{\left(q^{\nu+1}, q\right)_{\infty}}{(q, q)_{\infty}}\left(\frac{z}{2}\right)^{\nu}{ }_{0} \Phi_{1}\left(-; q^{\nu+1} ; q, \frac{z^{2} q^{\nu+1}}{4}\right)
\end{aligned}
$$

назовем модифицированными q-функииями Бесселя.

Очевидно,

$$
I_{\nu}^{(j)}(z, q)=e^{-i \nu \pi / 2} J_{\nu}^{(j)}\left(e^{i \pi / 2} z, q\right), \quad j=1,2 .
$$

В дальнейшем будем рассматривать функции

$$
\begin{aligned}
& I_{\nu}^{(1)}\left(\left(1-q^{2}\right) z ; q^{2}\right)=\sum_{k=0}^{\infty} \frac{\left(1-q^{2}\right)^{k}(z / 2)^{\nu+2 k}}{\left(q^{2}, q^{2}\right)_{k} \Gamma_{q^{2}}(\nu+k+1)}, \quad|z|<\frac{2}{1-q^{2}} \\
& I_{\nu}^{(2)}\left(\left(1-q^{2}\right) z ; q^{2}\right)=\sum_{k=0}^{\infty} \frac{q^{2 k(\nu+k)}\left(1-q^{2}\right)^{k}(z / 2)^{\nu+2 k}}{\left(q^{2}, q^{2}\right)_{k} \Gamma_{q^{2}}(\nu+k+1)} .
\end{aligned}
$$

Если $|q|<1$, ряд (18) сходится абсолютно для всех $z \neq 0$. Таким образом, $I_{\nu}^{(2)}\left(\left(1-q^{2}\right) z ; q^{2}\right)$ является голоморфной функцией вне окрестности нуля.

ЗАмЕЧАНИЕ 3.1. Для $I_{\nu}^{(j)}\left(\left(1-q^{2}\right) z ; q^{2}\right)$ имеем

$$
\lim _{q \rightarrow 1-0} I_{\nu}^{(j)}\left(\left(1-q^{2}\right) z ; q^{2}\right)=I_{\nu}(z), \quad j=1,2
$$




\section{2. Рекуррентные соотношения.}

ПРЕДЛОЖЕНИЕ 3.1. Функция $I_{\nu}^{(1)}\left(\left(1-q^{2}\right) z ; q^{2}\right)$ удовлетворяет соотношениям

$$
\begin{aligned}
& \frac{2}{(1+q) z} \partial_{q} z^{\nu} I_{-\nu}^{(1)}\left(\left(1-q^{2}\right) z ; q^{2}\right)=z^{\nu-1} I_{-\nu+1}^{(1)}\left(\left(1-q^{2}\right) z ; q^{2}\right), \\
& \frac{2}{(1+q) z} \partial_{q} z^{\nu} I_{\nu}^{(1)}\left(\left(1-q^{2}\right) z ; q^{2}\right)=z^{\nu-1} I_{\nu-1}^{(1)}\left(\left(1-q^{2}\right) z ; q^{2}\right),
\end{aligned}
$$

где оператор $\partial_{q}$ определяется формулой (5).

ДОКАЗАТЕЛЬСТво.

$$
\begin{aligned}
\frac{2}{(1+q) z} \partial_{q} z^{\nu} I_{-\nu}^{(1)}\left(\left(1-q^{2}\right) z ; q^{2}\right) & =\sum_{k=1}^{\infty} \frac{\left(1-q^{2}\right)^{k-1} z^{2 k-2}}{2^{-\nu+2 k-1}\left(q^{2}, q^{2}\right)_{k-1} \Gamma_{q^{2}}(-\nu+k+1)} \\
& =\sum_{k=0}^{\infty} \frac{\left(1-q^{2}\right)^{k} z^{2 k}}{2^{-\nu+1+2 k}\left(q^{2}, q^{2}\right)_{k} \Gamma_{q^{2}}(-\nu+1+k+1)} \\
& =z^{\nu-1} I_{-\nu+1}\left(\left(1-q^{2}\right) z ; q^{2}\right) .
\end{aligned}
$$

Формула (20) доказывается точно так же.

ПРЕДЛОЖЕНИЕ 3.2. Функция $I_{\nu}^{(1)}\left(\left(1-q^{2}\right) z ; q^{2}\right)$ удовлетворяет рекуррентным соотношениям

$$
\begin{aligned}
& I_{\nu-1}^{(1)}\left(\left(1-q^{2}\right) z ; q^{2}\right)-I_{\nu+1}^{(1)}\left(\left(1-q^{2}\right) z ; q^{2}\right) \\
& =\frac{2}{\left(1-q^{2}\right) z}\left(q^{-\nu}-q^{\nu}\right) I_{\nu}^{(1)}\left(\left(1-q^{2}\right) q z ; q^{2}\right), \\
& \begin{aligned}
& I_{\nu-1}^{(1)}\left(\left(1-q^{2}\right) z ; q^{2}\right)+I_{\nu+1}^{(1)}\left(\left(1-q^{2}\right) z ; q^{2}\right) \\
&= \frac{4}{\left(1-q^{2}\right) z} I_{\nu}^{(1)}\left(\left(1-q^{2}\right) z ; q^{2}\right) \\
& \quad-\frac{2}{\left(1-q^{2}\right) z}\left(q^{-\nu}+q^{\nu}\right) I_{\nu}^{(1)}\left(\left(1-q^{2}\right) q z ; q^{2}\right) .
\end{aligned}
\end{aligned}
$$

ДоКАЗАТЕЛЬСТво. Из (19) и (20) следует, что

$$
\begin{aligned}
& I_{\nu-1}^{(1)}\left(\left(1-q^{2}\right) z ; q^{2}\right)=\frac{2}{\left(1-q^{2}\right) z}\left(I_{\nu}^{(1)}\left(\left(1-q^{2}\right) z ; q^{2}\right)-q^{\nu} I_{\nu}^{(1)}\left(\left(1-q^{2}\right) q z ; q^{2}\right)\right) \\
& I_{\nu+1}^{(1)}\left(\left(1-q^{2}\right) z ; q^{2}\right)=\frac{2}{\left(1-q^{2}\right) z}\left(I_{\nu}^{(1)}\left(\left(1-q^{2}\right) z ; q^{2}\right)-q^{-\nu} I_{\nu}^{(1)}\left(\left(1-q^{2}\right) q z ; q^{2}\right)\right) .
\end{aligned}
$$

Складывая и вычитая эти равенства, получаем утверждение предложения. 
ПРЕДЛОЖЕНИЕ 3.3. Функция $I_{\nu}^{(2)}\left(\left(1-q^{2}\right) z ; q^{2}\right)$ удовлетворяет соотношениям

$$
\begin{aligned}
& \frac{2}{(1+q) z} \partial_{q} z^{\nu} I_{-\nu}^{(2)}\left(\left(1-q^{2}\right) z ; q^{2}\right)=q^{-\nu+1} z^{\nu-1} I_{-\nu+1}^{(2)}\left(\left(1-q^{2}\right) q z ; q^{2}\right) \\
& \frac{2}{(1+q) z} \partial_{q} z^{\nu} I_{\nu}^{(2)}\left(\left(1-q^{2}\right) z ; q^{2}\right)=q^{-\nu+1} z^{\nu-1} I_{\nu-1}^{(2)}\left(\left(1-q^{2}\right) q z ; q^{2}\right)
\end{aligned}
$$

ПРЕДЛОЖЕнИЕ 3.4. Функиия $I_{\nu}^{(2)}\left(\left(1-q^{2}\right) z ; q^{2}\right)$ удовлетворяет рекуррентныцм соотношениям

$$
\begin{aligned}
& q^{-\nu} I_{\nu-1}^{(2)}(\left.\left(1-q^{2}\right) z ; q^{2}\right)-q^{\nu} I_{\nu+1}^{(2)}\left(\left(1-q^{2}\right) z ; q^{2}\right) \\
&= \frac{2}{\left(1-q^{2}\right) z}\left(q^{-\nu}-q^{\nu}\right) I_{\nu}^{(2)}\left(\left(1-q^{2}\right) z ; q^{2}\right) \\
& q^{-\nu} I_{\nu-1}^{(2)}\left(\left(1-q^{2}\right) z ; q^{2}\right)+q^{\nu} I_{\nu+1}^{(2)}\left(\left(1-q^{2}\right) z ; q^{2}\right) \\
&=\frac{4}{\left(1-q^{2}\right) z} I_{\nu}^{(2)}\left(\left(1-q^{2}\right) q^{-1} z ; q^{2}\right) \\
& \quad-\frac{2}{\left(1-q^{2}\right) z}\left(q^{-\nu}+q^{\nu}\right) I_{\nu}^{(2)}\left(\left(1-q^{2}\right) z ; q^{2}\right) .
\end{aligned}
$$

Предложения 3.3 и 3.4 доказываются так же, как и предложения 3.1 и 3.2 .

\section{3. Разностные уравнения.}

ПРЕДЛОЖЕНИЕ 3.5. Функция $I_{\nu}^{(1)}\left(\left(1-q^{2}\right) z ; q^{2}\right)$ является решением разностного уравнения

$$
\left(1-\frac{q^{-2}\left(1-q^{2}\right)^{2}}{4} z^{2}\right) f\left(q^{-1} z\right)-\left(q^{-\nu}+q^{\nu}\right) f(z)+f(q z)=0
$$

ДокАЗАТЕЛЬСТво. Подставляя (17) в левую часть (23), получаем

$$
\begin{gathered}
\sum_{k=0}^{\infty}\left(q^{-\nu-2 k}-q^{-\nu}-q^{\nu}+q^{\nu+2 k}-\frac{q^{-\nu-2 k-2}\left(1-q^{2}\right)^{2} z^{2}}{4}\right) \frac{\left(1-q^{2}\right)^{k}(z / 2)^{\nu+2 k}}{\left(q^{2}, q^{2}\right)_{k} \Gamma_{q^{2}}(\nu+k+1)} \\
=\sum_{k=1}^{\infty} \frac{q^{-\nu-2 k}\left(1-q^{2 k}\right)\left(1-q^{2 \nu+2 k}\right)\left(1-q^{2}\right)^{k}(z / 2)^{\nu+2 k}}{\left(q^{2}, q^{2}\right)_{k} \Gamma_{q^{2}}(\nu+k+1)} \\
-\sum_{k=0}^{\infty} \frac{q^{-\nu-2 k-2}\left(1-q^{2}\right)^{k+2}(z / 2)^{\nu+2 k+2}}{\left(q^{2}, q^{2}\right)_{k} \Gamma_{q^{2}}(\nu+k+1)} .
\end{gathered}
$$

Меняя $k$ на $k+1$ в первом слагаемом, получаем нуль.

СлЕДСТвиЕ 3.1. Функция $I_{-\nu}^{(1)}\left(\left(1-q^{2}\right) z ; q^{2}\right)$ удовлетворяет уравнению (23).

ДоказАтЕльство. Очевидно, левая часть (23) - четная функция $\nu$. Тогда замена $\nu$ на $-\nu$ в (17) переводит решение уравнения (23) в решение того же уравнения. 
ОПРЕДЕЛЕНИЕ 3.2. q-вронскиан двух решений $f_{\nu}^{1}(z), f_{\nu}^{2}(z)$ разностного уравнения второго порядка определим следующим образом

$$
W\left(f_{\nu}^{1}, f_{\nu}^{2}\right)(z)=f_{\nu}^{1}(z) f_{\nu}^{2}(q z)-f_{\nu}^{1}(q z) f_{\nu}^{2}(z) .
$$

Если $q$-вронскиан не обращается в нуль, любое решение разностного уравнения второго порядка может быть записано в виде

$$
f_{\nu}(z)=C_{1} f_{\nu}^{1}(z)+C_{2} f_{\nu}^{2}(z) .
$$

В этом случае функции $f_{\nu}^{1}(z)$ и $f_{\nu}^{2}(z)$ образуют фундаментальную систему решений данного уравнения.

ПрЕДЛОЖЕНИЕ 3.6. Если $\nu$ не является целым числом, функиии $I_{\nu}^{(1)}\left(\left(1-q^{2}\right) z ; q^{2}\right)$ и $I_{-\nu}^{(1)}\left(\left(1-q^{2}\right) z ; q^{2}\right)$ образуют фундаментальную систему решений уравнения $(23)\left(z \neq \pm 2 q^{-r} /\left(1-q^{2}\right), r=0,1, \ldots\right)$.

ДоКАЗАТЕЛЬСТво. Рассмотрим $q$-вронскиан

$$
\begin{aligned}
W\left(I_{\nu}^{(1)}, I_{-\nu}^{(1)}\right)(z)=I_{\nu}^{(1)}\left(\left(1-q^{2}\right)\right. & \left.z ; q^{2}\right) I_{-\nu}^{(1)}\left(\left(1-q^{2}\right) q z ; q^{2}\right) \\
& -I_{\nu}^{(1)}\left(\left(1-q^{2}\right) q z ; q^{2}\right) I_{-\nu}^{(1)}\left(\left(1-q^{2}\right) z ; q^{2}\right) .
\end{aligned}
$$

Так как $I_{\nu}^{(1)}$ и $I_{-\nu}^{(1)}$ являются решениями $(23)$, то

$$
\begin{aligned}
I_{ \pm \nu}^{(1)}\left(\left(1-q^{2}\right) q^{2} z ; q^{2}\right)=-\left(1-\frac{\left(1-q^{2}\right)^{2}}{4} z^{2}\right) & I_{ \pm \nu}^{(1)}\left(\left(1-q^{2}\right) z ; q^{2}\right) \\
& +\left(q^{-\nu}+q^{\nu}\right) I_{ \pm \nu}^{(1)}\left(\left(1-q^{2}\right) q z ; q^{2}\right)
\end{aligned}
$$

Таким образом,

$$
\begin{aligned}
W\left(I_{\nu}^{(1)}, I_{-\nu}^{(1)}\right)(q z)= & I_{\nu}^{(1)}\left(\left(1-q^{2}\right) q z ; q^{2}\right) I_{-\nu}^{(1)}\left(\left(1-q^{2}\right) q^{2} z ; q^{2}\right) \\
& -I_{\nu}^{(1)}\left(\left(1-q^{2}\right) q^{2} z ; q^{2}\right) I_{-\nu}^{(1)}\left(\left(1-q^{2}\right) q z ; q^{2}\right) \\
= & \left(1-\frac{\left(1-q^{2}\right)^{2}}{4} z^{2}\right) W\left(I_{\nu}^{(1)}, I_{-\nu}^{(1)}\right)(z) .
\end{aligned}
$$

Сравнивая это равенство с (8), выводим

$$
W\left(I_{\nu}^{(1)}, I_{-\nu}^{(1)}\right)(z)=C_{\nu} e_{q^{2}}\left(\frac{\left(1-q^{2}\right)^{2}}{4} z^{2}\right) .
$$

Полагая здесь и в $(24) z=0$, получаем

$$
C_{\nu}=\frac{q^{-\nu}\left(1-q^{2}\right)}{\Gamma_{q^{2}}(\nu) \Gamma_{q^{2}}(1-\nu)} .
$$

Таким образом, окончательно

$$
W\left(I_{\nu}^{(1)}, I_{-\nu}^{(1)}\right)(z)=\frac{q^{-\nu}\left(1-q^{2}\right)}{\Gamma_{q^{2}}(\nu) \Gamma_{q^{2}}(1-\nu)} e_{q^{2}}\left(\frac{\left(1-q^{2}\right)^{2}}{4} z^{2}\right) .
$$

Очевидно, эта функция определена при $z \neq \pm 2 q^{-r} /\left(1-q^{2}\right), r=0,1, \ldots$, и не обрашается в нуль.

Если $\nu=n$-целое число, то из (17) и (18)

$$
I_{-n}^{(j)}\left(\left(1-q^{2}\right) z ; q^{2}\right)=I_{n}^{(j)}\left(\left(1-q^{2}\right) z ; q^{2}\right), \quad j=1,2 .
$$


ПрЕДЛОЖЕНИЕ 3.7. Функиия $I_{\nu}^{(2)}\left(\left(1-q^{2}\right) z ; q^{2}\right)$ является решением уравнения

$$
f\left(q^{-1} z\right)-\left(q^{-\nu}+q^{\nu}\right) f(z)+\left(1-\frac{\left(1-q^{2}\right)^{2}}{4} z^{2}\right) f(q z)=0 .
$$

Это предложение доказывается так же, как и предложение 3.5 .

СлЕДСТВИЕ 3.2. Функции (17) и (18) связаны соотношением

$$
I_{\nu}^{(1)}\left(\left(1-q^{2}\right) z ; q^{2}\right)=e_{q^{2}}\left(\frac{\left(1-q^{2}\right)^{2}}{4} z^{2}\right) I_{\nu}^{(2)}\left(\left(1-q^{2}\right) z ; q^{2}\right) .
$$

ДокАЗАТЕЛЬСтво. Из (27) и (8) следует, что функция

$$
e_{q^{2}}\left(\frac{\left(1-q^{2}\right)^{2}}{4} z^{2}\right) I_{\nu}^{(2)}\left(\left(1-q^{2}\right) z ; q^{2}\right)
$$

удовлетворяет уравнению (23). Значит, если $\nu$ не является целым,

$$
\begin{aligned}
e_{q^{2}}\left(\frac{\left(1-q^{2}\right)^{2}}{4} z^{2}\right) I_{\nu}^{(2)}\left(\left(1-q^{2}\right) z ; q^{2}\right) & \\
& =A I_{\nu}^{(1)}\left(\left(1-q^{2}\right) z ; q^{2}\right)+B I_{-\nu}^{(1)}\left(\left(1-q^{2}\right) z ; q^{2}\right) .
\end{aligned}
$$

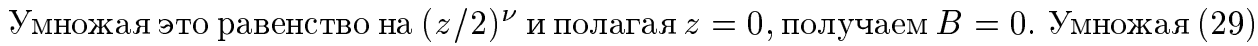
на $(z / 2)^{-\nu}$ и полагая $z=0$, получаем $A=1$.

Так как (17) и (18) - непрерывные функции $\nu$, соотношение (28) верно и для целых значений $\nu=n$.

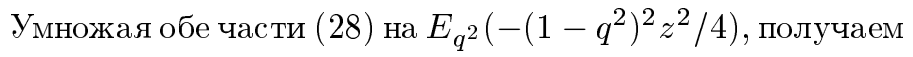

СлеДСтвИЕ 3.3. Справедливо равенство

$$
I_{\nu}^{(2)}\left(\left(1-q^{2}\right) z ; q^{2}\right)=E_{q^{2}}\left(-\frac{\left(1-q^{2}\right)^{2}}{4} z^{2}\right) I_{\nu}^{(1)}\left(\left(1-q^{2}\right) z ; q^{2}\right) .
$$

ПРЕДЛОЖЕНИЕ 3.8. Если $\nu$ не является целымм числом, функции $I_{\nu}^{(2)}\left(\left(1-q^{2}\right) z ; q^{2}\right)$ и $I_{-\nu}^{(2)}\left(\left(1-q^{2}\right) z ; q^{2}\right)$ образуют фундаментальную систему решений уравнения (27) при $z \neq \pm 2 q^{-r} /\left(1-q^{2}\right), r=0,1, \ldots$.

ДоКАЗАТЕЛЬСТво. Из (30) и (25) следует, что

$$
\begin{aligned}
W\left(I_{\nu}^{(2)}, I_{-\nu}^{(2)}\right)(z) & =E_{q^{2}}^{2}\left(-\frac{\left(1-q^{2}\right)^{2}}{4} z^{2}\right) W\left(I_{\nu}^{(1)}, I_{-\nu}^{(1)}\right)(z) \\
& =\frac{q^{-\nu}\left(1-q^{2}\right)}{\Gamma_{q^{2}}(\nu) \Gamma_{q^{2}}(1-\nu)} E_{q^{2}}\left(-\frac{\left(1-q^{2}\right)^{2}}{4} z^{2}\right) .
\end{aligned}
$$

Очевидно, эта функция не обращается в нуль при $z \neq \pm 2 q^{-r} /\left(1-q^{2}\right), r=0,1, \ldots$.

ПРЕДЛОЖЕНИЕ 3.9. Функция $I_{\nu}^{(1)}\left(\left(1-q^{2}\right) z ; q^{2}\right)$ вне окрестности нуля является мероморфной функиией $c$ простыми полюсами в точках $z= \pm 2 q^{-r} /\left(1-q^{2}\right), r=0,1, \ldots$

ДокАЗАТЕЛЬСТво. Утверждение предложения следует из (28) и замечания 2.1.

ЗАмЕчАниЕ 3.2 . Если $q \rightarrow 1-0$, все полюсы функции $I_{\nu}^{(1)}\left(\left(1-q^{2}\right) z ; q^{2}\right)$

$$
z_{r}= \pm \frac{2 q^{-r}}{1-q^{2}}, \quad r=0,1, \ldots
$$

стремятся к бесконечности вдоль вещественной оси. 


\section{§4. Ряды лорановского типа для $q-\mathrm{МФБ}$}

$\mathrm{K}$ сожалению, функция $I_{\nu}^{(1)}\left(\left(1-q^{2}\right) z ; q^{2}\right)$ определяется степенным рядом (17) лишь в области $|z|<2 /\left(1-q^{2}\right)$, в то время, как нам нужно представление этой функции в виде ряда на всей комплексной плоскости. Здесь мы попытаемся исправить ситуацию.

ПРЕДЛОЖЕНИЕ 4.1. Любое решение уравнения (23) может быть записано в виде

$$
\begin{aligned}
f_{\nu}(z)= & \frac{a}{\sqrt{z}} e_{q}\left(\frac{1-q^{2}}{2} z\right){ }_{2} \Phi_{1}\left(q^{\nu+1 / 2}, q^{-\nu+1 / 2} ;-q ; q, \frac{2 q}{\left(1-q^{2}\right) z}\right) \\
& +\frac{b}{\sqrt{z}} e_{q}\left(-\frac{1-q^{2}}{2} z\right){ }_{2} \Phi_{1}\left(q^{\nu+1 / 2}, q^{-\nu+1 / 2} ;-q ; q,-\frac{2 q}{\left(1-q^{2}\right) z}\right),
\end{aligned}
$$

где ${ }_{2} \Phi_{1}$ определяется формулой (16).

ДокАЗАТЕльство. Пусть $f_{\nu}(z)$ - решение уравнения (23). Представим ее в виде

$$
f_{\nu}(z)=\frac{a}{\sqrt{z}} e_{q}\left(\frac{1-q^{2}}{2} z\right) \varphi_{\nu}^{1}(z)+\frac{b}{\sqrt{z}} e_{q}\left(-\frac{1-q^{2}}{2} z\right) \varphi_{\nu}^{2}(z) .
$$

Предположим,что первое слагаемое в правой части удовлетворяет уравнению (23). Тогда второе слагаемое также является его решением. Рассмотрим первое слагаемое. Подставляя его в (23) и используя (6), (7), получаем разностное уравнение для $\varphi_{\nu}^{1}(z)$

$$
\left(1+\frac{q^{-1}\left(1-q^{2}\right)}{2} z\right) q \varphi_{\nu}^{1}\left(q^{-1} z\right)-q^{1 / 2}\left(q^{-\nu}+q^{\nu}\right) \varphi_{\nu}^{1}(z)+\left(1-\frac{1-q^{2}}{2} z\right) \varphi_{\nu}^{1}(q z)=0 .
$$

Представим функцию $\varphi_{\nu}^{1}(z)$ в виде

$$
\varphi_{\nu}^{1}(z)=\sum_{k=0}^{\infty} a_{k} z^{-k}
$$

Тогда $a_{0}$ произвольно, для любого $k \geqslant 1$

$$
a_{k}=a_{k-1} \frac{2 q\left(1-q^{\nu-1 / 2+k}\right)\left(1-q^{-\nu-1 / 2+k}\right)}{\left(1-q^{2}\right)\left(1-q^{2 k}\right)}
$$

и

$$
a_{k}=a_{0} \frac{2^{k} q^{k}\left(q^{\nu+1 / 2}, q\right)_{k}\left(q^{-\nu+1 / 2}, q\right)_{k}}{\left(1-q^{2}\right)^{k}\left(q^{2}, q^{2}\right)_{k}} .
$$

Полагая $a_{0}=1$, получаем

$$
\varphi_{\nu}^{1}(z)=\sum_{k=0}^{\infty} \frac{2^{k} q^{k}\left(q^{\nu+1 / 2}, q\right)_{k}\left(q^{-\nu+1 / 2}, q\right)_{k}}{\left(1-q^{2}\right)^{k}\left(q^{2}, q^{2}\right)_{k}} z^{-k}
$$


Точно так же

$$
\varphi_{\nu}^{2}(z)=\sum_{k=0}^{\infty}(-1)^{k} \frac{2^{k} q^{k}\left(q^{\nu+1 / 2}, q\right)_{k}\left(q^{-\nu+1 / 2}, q\right)_{k}}{\left(1-q^{2}\right)^{k}\left(q^{2}, q^{2}\right)_{k}} z^{-k}=\varphi_{\nu}^{1}(-z) .
$$

Ввиду этого, мы в дальнейшем не будем писать верный индекс. Ряд (34) абсолютно сходится при $|z|>2 q /\left(1-q^{2}\right)$.

Коэффициенты $a$ и $b$ по функции $f_{\nu}(z)$ определяются однозначно. Действительно, пусть при $z=z_{0},\left|z_{0}\right|>2 /\left(1-q^{2}\right), f_{\nu}\left(z_{0}\right)=A$ и $f_{\nu}\left(q z_{0}\right)=B$. Используя (6), (7), получаем систему уравнений для определения $a$ и $b$

$$
\left\{\begin{array}{c}
\frac{a}{\sqrt{z_{0}}} e_{q}\left(\frac{\left(1-q^{2}\right)}{2} z_{0}\right) \varphi_{\nu}\left(z_{0}\right)+\frac{b}{\sqrt{z_{0}}} e_{q}\left(-\frac{\left(1-q^{2}\right)}{2} z_{0}\right) \varphi_{\nu}\left(-z_{0}\right)=A \\
\frac{a}{\sqrt{q z_{0}}}\left(1-\frac{\left(1-q^{2}\right)}{2} z_{0}\right) e_{q}\left(\frac{\left(1-q^{2}\right)}{2} z_{0}\right) \varphi_{\nu}\left(q z_{0}\right) \\
\quad+\frac{b}{\sqrt{q z_{0}}}\left(1+\frac{\left(1-q^{2}\right)}{2} z_{0}\right) e_{q}\left(-\frac{\left(1-q^{2}\right)}{2} z_{0}\right) \varphi_{\nu}\left(-q z_{0}\right)=B
\end{array}\right.
$$

Ее определитель имеет вид

$$
\begin{aligned}
W=\frac{q^{-1 / 2}}{z_{0}} e_{q^{2}}\left(\frac{\left(1-q^{2}\right)^{2}}{4}\right. & \left.z^{2}\right)\left(\varphi_{\nu}\left(z_{0}\right) \varphi_{\nu}\left(-q z_{0}\right)-\varphi_{\nu}\left(q z_{0}\right) \varphi_{\nu}\left(-z_{0}\right)\right. \\
& \left.+\frac{1-q^{2}}{2} z_{0}\left(\varphi_{\nu}\left(z_{0}\right) \varphi_{\nu}\left(-q z_{0}\right)+\varphi_{\nu}\left(q z_{0}\right) \varphi_{\nu}\left(-z_{0}\right)\right)\right)
\end{aligned}
$$

Предположим, что $W\left(z_{0}\right)=0$ для некоторого $z_{0},\left|z_{0}\right|>2 /\left(1-q^{2}\right)$. Тогда

$$
\left\{\begin{array}{l}
\varphi_{\nu}\left(z_{0}\right) \varphi_{\nu}\left(-q z_{0}\right)-\varphi_{\nu}\left(q z_{0}\right) \varphi_{\nu}\left(-z_{0}\right)=0 \\
\varphi_{\nu}\left(z_{0}\right) \varphi_{\nu}\left(-q z_{0}\right)+\varphi_{\nu}\left(q z_{0}\right) \varphi_{\nu}\left(-z_{0}\right)=0
\end{array}\right.
$$

или

$$
\left\{\begin{array}{l}
\varphi_{\nu}\left(z_{0}\right) \varphi_{\nu}\left(-q z_{0}\right)=0 \\
\varphi_{\nu}\left(q z_{0}\right) \varphi_{\nu}\left(-z_{0}\right)=0 .
\end{array}\right.
$$

Из $(34)$ следует, что $\varphi_{\nu}\left(z_{0}\right)=\varphi_{\nu}\left(q z_{0}\right)=0$ (или $\varphi_{\nu}\left(-z_{0}\right)=\varphi_{\nu}\left(-q z_{0}\right)=0$ ) удовлетворяет (36). В этом случае из $(33)$ имеем $\varphi_{\nu}\left(q^{-1} z_{0}\right)=0$, и, следовательно, $\varphi_{\nu}\left(q^{-r} z_{0}\right)=0$ для любого $r=0,1, \ldots$ Но это противоречит очевидному равенству $\lim _{|z| \rightarrow \infty} \varphi_{\nu}(z)=1$. Таким образом, $W \neq 0$, и $a, b$ определяются однозначно из (35).

Из (16) легко видеть, что

$$
\begin{aligned}
\varphi_{\nu}(z) & =\sum_{k=0}^{\infty} \frac{\left(q^{\nu+1 / 2}, q\right)_{k}\left(q^{-\nu+1 / 2}, q\right)_{k}}{(q, q)_{k}(-q, q)_{k}}\left(\frac{2 q}{\left(1-q^{2}\right) z}\right)^{k} \\
& ={ }_{2} \Phi_{1}\left(q^{\nu+1 / 2}, q^{-\nu+1 / 2} ;-q ; q, \frac{2 q}{\left(1-q^{2}\right) z}\right) .
\end{aligned}
$$

Подставляя эту функцию в (32), получаем (31).

Для краткости обозначим

$$
{ }_{2} \Phi_{1}\left(q^{\nu+1 / 2}, q^{-\nu+1 / 2} ;-q ; q, \frac{2 q}{\left(1-q^{2}\right) z}\right)=\Phi_{\nu}(z) .
$$


ПРЕДЛОЖЕНИЕ 4.2. $q$-МФБ $I_{\nu}^{(1)}\left(\left(1-q^{2}\right) z ; q^{2}\right)$ nри $z \neq 0$ может быть представлена в виде

$$
I_{\nu}^{(1)}\left(\left(1-q^{2}\right) z ; q^{2}\right)=\frac{a_{\nu}}{\sqrt{z}}\left(e_{q}\left(\frac{1-q^{2}}{2} z\right) \Phi_{\nu}(z)+i e^{i \nu \pi} e_{q}\left(-\frac{1-q^{2}}{2} z\right) \Phi_{\nu}(-z)\right),
$$

где $\Phi_{\nu}(z)$ определяется формулой (37) и

$$
a_{\nu}=\sqrt{\frac{2}{1-q^{2}}} e_{q}(-1) \frac{I_{\nu}^{(2)}\left(2 ; q^{2}\right)}{\Phi_{\nu}\left(\frac{2}{1-q^{2}}\right)} .
$$

ДоКАЗАТЕЛЬСТво. Из следствия 3.2 и предложения 4.1 следует, что

$$
\begin{aligned}
e_{q}\left(\frac{1-q^{2}}{2} z\right) e_{q}(- & \left.\frac{1-q^{2}}{2} z\right) I_{\nu}^{(2)}\left(\left(1-q^{2}\right) z ; q^{2}\right) \\
& =\frac{a_{\nu}}{\sqrt{z}} e_{q}\left(\frac{1-q^{2}}{2} z\right) \Phi_{\nu}(z)+\frac{b_{\nu}}{\sqrt{z}} e_{q}\left(-\frac{1-q^{2}}{2} z\right) \Phi_{\nu}(-z)
\end{aligned}
$$

в области $2 q /\left(1-q^{2}\right)<|z|<2 /\left(1-q^{2}\right)$. Функции, стоящие в обеих частях этого равенства, являются мероморфными в области $z \neq 0$ и имеют простые полюсы в точках $z= \pm 2 q^{-r} /\left(1-q^{2}\right), r=0,1, \ldots$ В силу единственности аналитического продолжения равенство (40) остается справедливым в области $z \neq 0$. Потребуем, чтобы совпадали вычеты в полюсах в обеих частях (40). Тогда при $z=2 q^{-r} /\left(1-q^{2}\right)$

$$
e_{q}\left(-q^{-r}\right) I_{\nu}^{(2)}\left(2 q^{-r} ; q^{2}\right)=a_{\nu} q^{r / 2} \sqrt{\frac{1-q^{2}}{2}} \Phi_{\nu}\left(\frac{2 q^{-r}}{1-q^{2}}\right)
$$

и при $z=-2 q^{-r} /\left(1-q^{2}\right)$

$$
e_{q}\left(-q^{-r}\right) I_{\nu}^{(2)}\left(-2 q^{-r} ; q^{2}\right)=b_{\nu} \frac{q^{r / 2}}{i} \sqrt{\frac{1-q^{2}}{2}} \Phi_{\nu}\left(-\frac{2 q^{-r}}{1-q^{2}}\right) .
$$

Из (18) следует, что

$$
I_{\nu}^{(2)}\left(-2 q^{-r} ; q^{2}\right)=e^{i \nu \pi} I_{\nu}^{(2)}\left(2 q^{-r} ; q^{2}\right) .
$$

Следовательно, из (41) и (42)

$$
b_{\nu}=i e^{i \nu \pi} a_{\nu} .
$$

Полагая $r=0$ в (41), получаем (39). Подставляя (43) в (40), выводим утверждение предложения.

ПРЕДЛОЖЕНИЕ 4.3. Коэффициенты $a_{\nu}$ (39) удовлетворяют рекуррентному соотношению

$$
a_{\nu+1}=a_{\nu} q^{-\nu-1 / 2}
$$

и условию

$$
a_{\nu} a_{-\nu}=\frac{q^{-\nu+1 / 2}}{2 \Gamma_{q^{2}}(\nu) \Gamma_{q^{2}}(1-\nu) \sin \nu \pi}
$$


ДокАЗАТЕЛЬСТво. Подставим (38) в (21) и (22). Тогда из (6) имеем

$$
\begin{aligned}
a_{\nu-1} \Phi_{\nu-1}(z)-a_{\nu+1} \Phi_{\nu+1}(z)= & 2 a_{\nu} q^{-1 / 2} \frac{q^{-\nu}-q^{\nu}}{\left(1-q^{2}\right) z}\left(1-\frac{1-q^{2}}{2} z\right) \Phi_{\nu}(q z), \\
a_{\nu-1} \Phi_{\nu-1}(z)+a_{\nu+1} \Phi_{\nu+1}(z)= & \frac{4 a_{\nu}}{\left(1-q^{2}\right) z} \Phi_{\nu}(z) \\
& -2 a_{\nu} q^{-1 / 2} \frac{q^{-\nu}+q^{\nu}}{\left(1-q^{2}\right) z}\left(1-\frac{1-q^{2}}{2} z\right) \Phi_{\nu}(q z) .
\end{aligned}
$$

Устремляя $z$ к бесконечности в (46) и (47), приходим к системе уравнений

$$
\left\{\begin{array}{l}
a_{\nu-1}-a_{\nu+1}=-a_{\nu} q^{-1 / 2}\left(q^{-\nu}-q^{\nu}\right) ; \\
a_{\nu-1}+a_{\nu+1}=a_{\nu} q^{-1 / 2}\left(q^{-\nu}+q^{\nu}\right) .
\end{array}\right.
$$

Из данной системы получаем первое утверждение предложения.

Рассмотрим $q$-вронскиан $W\left(I_{\nu}^{(1)}, I_{-\nu}^{(1)}\right)(25)$ и представление (38). Тогда при $\nu \neq n$

$$
\begin{aligned}
& \frac{q^{-\nu}\left(1-q^{2}\right)}{\Gamma_{q^{2}}(\nu) \Gamma_{q^{2}}(1-\nu)} e_{q^{2}}\left(\frac{\left(1-q^{2}\right)^{2}}{4} z^{2}\right)=q^{-1 / 2} a_{\nu} a_{-\nu} z^{-1} e_{q}\left(\frac{1-q^{2}}{2} z\right) e_{q}\left(-\frac{1-q^{2}}{2} z\right) \\
& \quad \times\left(i e^{-i \nu \pi}\left(\left(1+\frac{1-q^{2}}{2} z\right) \Phi_{\nu}(z) \Phi_{\nu}(-q z)-\left(1-\frac{1-q^{2}}{2} z\right) \Phi_{\nu}(q z) \Phi_{\nu}(-z)\right)\right. \\
& \left.\quad+i e^{i \nu \pi}\left(\left(1-\frac{1-q^{2}}{2} z\right) \Phi_{\nu}(-z) \Phi_{\nu}(q z)-\left(1+\frac{1-q^{2}}{2} z\right) \Phi_{\nu}(-q z) \Phi_{\nu}(z)\right)\right)
\end{aligned}
$$

Разделив это равенство на $q$-экспоненты и устремив $z$ к бесконечности, получим (45).

Если $\nu=n$, то из (44) имеем

$$
a_{n}=a_{n-k} q^{-k / 2(2 n-k)}
$$

Положим $k=2 n$. Тогда $a_{n}=a_{-n}$.

Из $(45)$ и $[12,1.10 .16]$ имеем

$$
a_{n}^{2}=\frac{q^{-n^{2}+1 / 2} \ln q^{-2}}{2 \pi\left(1-q^{2}\right)}
$$

ПРЕДЛОЖЕнИЕ 4.4. $q-М \Phi Б I_{\nu}^{(2)}\left(\left(1-q^{2}\right) z ; q^{2}\right)$ nри $z \neq 0$ может бъть представлена в виде

$$
I_{\nu}^{(2)}\left(\left(1-q^{2}\right) z ; q^{2}\right)=\frac{a_{\nu}}{\sqrt{z}}\left(E_{q}\left(\frac{1-q^{2}}{2} z\right) \Phi_{\nu}(z)+i e^{i \nu \pi} E_{q}\left(-\frac{1-q^{2}}{2} z\right) \Phi_{\nu}(-z)\right) .
$$

ДокАЗАТЕЛЬСТво. Это утверждение следует из следствия 3.3 и (3). 


\section{§5. q-функции Макдональда}

В классическом анализе функция Макдональда определяется следующим образом

$$
K_{\nu}(z)=\frac{\pi}{2 \sin \nu \pi}\left(I_{-\nu}(z)-I_{\nu}(z)\right)
$$

для $\nu \neq n$, для $\nu=n$ - предельным переходом в (50) при $\nu \rightarrow n$. Здесь мы проведем корректное "квантование" этого определения таким образом, чтобы были "проквантованы" и все свойства данной функции.

Лемма 5.1. Для $a_{\nu}$ (39) имеет место формула

$$
\left.\left(\frac{\partial}{\partial \nu} a_{\nu}-\frac{\partial}{\partial \nu} a_{-\nu}\right)\right|_{\nu=n}=a_{n} \widetilde{a}
$$

əдe

$$
\tilde{a}=\frac{2 \ln q^{2}}{I_{0}^{(2)}\left(2 ; q^{2}\right)} \sum_{k=0}^{\infty} \frac{q^{2 k^{2}}}{\left(q^{2}, q^{2}\right)_{k}^{2}}\left(k-\sum_{l=1}^{\infty} \frac{q^{2 l+2 k+2}}{1-q^{2 l+2 k+2}}\right) .
$$

ДокАЗАТЕЛЬСТво. Пусть $n=[\nu]$. Из (44) следует, что

$$
a_{\nu}=a_{\nu-n} q^{-n \nu+n^{2} / 2}, \quad a_{-\nu}=a_{-\nu+n} q^{-n \nu+n^{2} / 2} .
$$

Тогда

$$
\left.\left(\frac{\partial}{\partial \nu} a_{\nu}-\frac{\partial}{\partial \nu} a_{-\nu}\right)\right|_{\nu=n}=\left.\left(\frac{\partial}{\partial \nu} a_{\nu-n}-\frac{\partial}{\partial \nu} a_{-\nu+n}\right)\right|_{\nu=n} q^{-n^{2} / 2}
$$

Используя (39), получаем

$$
\begin{aligned}
& \left.\left(\frac{\partial}{\partial \nu} a_{\nu}-\frac{\partial}{\partial \nu} a_{-\nu}\right)\right|_{\nu=0} \\
& \quad=\left.\frac{\sqrt{2 /\left(1-q^{2}\right)} e_{q}(-1) I_{0}^{(2)}\left(2 ; q^{2}\right)}{\Phi_{0}\left(2 /\left(1-q^{2}\right)\right)} \frac{q^{-n^{2} / 2}}{I_{0}^{(2)}\left(2 ; q^{2}\right)}\left(\frac{\partial}{\partial \nu} I_{\nu}^{(2)}\left(2 ; q^{2}\right)-\frac{\partial}{\partial \nu} I_{-\nu}^{(2)}\left(2 ; q^{2}\right)\right)\right|_{\nu=0} \\
& \quad=\left.a_{0} \frac{q^{-\frac{n^{2}}{2}}}{I_{0}^{(2)}\left(2 ; q^{2}\right)}\left(\frac{\partial}{\partial \nu} I_{\nu}^{(2)}\left(2 ; q^{2}\right)-\frac{\partial}{\partial \nu} I_{-\nu}^{(2)}\left(2 ; q^{2}\right)\right)\right|_{\nu=0}
\end{aligned}
$$

Из (18) следует, что

$$
\begin{aligned}
\frac{\partial}{\partial \nu} I_{\nu}^{(2)}\left(2 ; q^{2}\right)= & \ln q^{2} \sum_{k=0}^{\infty} \frac{k q^{2 k(\nu+k)}}{\left(q^{2}, q^{2}\right)_{k}\left(1-q^{2}\right)^{\nu+k} \Gamma_{q^{2}}(\nu+k+1)} \\
& -\ln \left(1-q^{2}\right) I_{\nu}^{(2)}\left(2 ; q^{2}\right)-\sum_{k=0}^{\infty} \frac{q^{2 k(\nu+k)} \psi_{q^{2}}(\nu+k+1)}{\left(q^{2}, q^{2}\right)_{k}\left(1-q^{2}\right)^{\nu+k} \Gamma_{q^{2}}(\nu+k+1)} .
\end{aligned}
$$


Из (12) и (13)

$$
\begin{aligned}
& \left.\left(\frac{\partial}{\partial \nu} I_{\nu}^{(2)}\left(2 ; q^{2}\right)-\frac{\partial}{\partial \nu} I_{-\nu}^{(2)}\left(2 ; q^{2}\right)\right)\right|_{\nu=0} \\
& \quad=2 \ln q^{2} \sum_{k=0}^{\infty} \frac{k q^{2 k^{2}}}{\left(q^{2}, q^{2}\right)_{k}^{2}}-2 \ln \left(1-q^{2}\right) I_{0}^{(2)}\left(2 ; q^{2}\right)-2 \sum_{k=0}^{\infty} \frac{q^{2 k^{2}}}{\left(q^{2}, q^{2}\right)_{k}^{2}} \psi_{q^{2}}(k+1) \\
& \quad=2 \ln q^{2} \sum_{k=0}^{\infty} \frac{k q^{2 k^{2}}}{\left(q^{2}, q^{2}\right)_{k}^{2}}-2 \ln q^{2} \sum_{k=0}^{\infty} \frac{q^{2 k^{2}}}{\left(q^{2}, q^{2}\right)_{k}^{2}} \sum_{l=1}^{\infty} \frac{q^{2 l+2 k+2}}{1-q^{2} l+2 k+2} .
\end{aligned}
$$

Теперь (51) следует из (52) и (53).

ОПРЕДЕЛЕНИЕ 5.1. q-функиию Макдональда (q-ФМ) определим следующим образом

$$
\begin{aligned}
& K_{\nu}^{(j)}\left(\left(1-q^{2}\right) z ; q^{2}\right) \\
& \quad=\frac{q^{-\nu^{2}+1 / 2}}{4\left(a_{\nu} a_{-\nu}\right)^{3 / 2} \sin \nu \pi}\left(a_{\nu} I_{-\nu}^{(j)}\left(\left(1-q^{2}\right) z ; q^{2}\right)-a_{-\nu} I_{\nu}^{(j)}\left(\left(1-q^{2}\right) z ; q^{2}\right)\right),
\end{aligned}
$$

где $a_{\nu}$ определяется формулой $(39), j=1,2$.

Как и в классическом случае, это определение должно быть распространено на целые значения индекса $\nu=n$. Рассмотрим предел (54) для $j=1$, принимая во внимание (48),

$$
\begin{aligned}
& K_{n}^{(1)}\left(\left(1-q^{2}\right) z ; q^{2}\right) \\
& \qquad=\frac{q^{-n^{2}+1 / 2}}{4 a_{n}^{3}} \lim _{\nu \rightarrow n} \frac{a_{\nu} I_{-\nu}^{(1)}\left(\left(1-q^{2}\right) z ; q^{2}\right)-a_{-\nu} I_{\nu}^{(1)}\left(\left(1-q^{2}\right) z ; q^{2}\right)}{\sin \nu \pi} .
\end{aligned}
$$

Используя (17), можем написать

$$
\begin{aligned}
\frac{\partial}{\partial \nu} I_{\nu}^{(1)}((1 & \left.\left.-q^{2}\right) z ; q^{2}\right) \\
& =\ln (z / 2) I_{\nu}^{(1)}\left(\left(1-q^{2}\right) z ; q^{2}\right)-\sum_{k=0}^{\infty} \frac{\left(1-q^{2}\right)^{k}(z / 2)^{\nu+k} \psi_{q^{2}}(\nu+k+1)}{\left(q^{2}, q^{2}\right)_{k} \Gamma_{q^{2}}(\nu+k+1)}
\end{aligned}
$$

Ввиду следствия 2.1

$$
\begin{aligned}
& \left.\left(\frac{\partial}{\partial \nu} I_{-\nu}^{(1)}\left(\left(1-q^{2}\right) z ; q^{2}\right)-\frac{\partial}{\partial \nu} I_{\nu}^{(1)}\left(\left(1-q^{2}\right) z ; q^{2}\right)\right)\right|_{\nu=n}=-2 \ln (z / 2) I_{n}^{(1)}\left(\left(1-q^{2}\right) z ; q^{2}\right) \\
& +\ln q^{2} \sum_{k=0}^{n-1}(-1)^{n-k-1} \frac{q^{-(n-k-1)(n-k-2)}\left(1-q^{2}\right)^{-n+2 k}\left(q^{2}, q^{2}\right)_{n-k-1}}{\left(q^{2}, q^{2}\right)_{k}}(z / 2)^{-n+2 k} \\
& +2 \sum_{k=0}^{\infty} \frac{\left(1-q^{2}\right)^{n+2 k}(z / 2)^{n+2 k}}{\left(q^{2}, q^{2}\right)_{k}\left(q^{2}, q^{2}\right)_{n+k}}\left(\psi_{q^{2}}(n+k+1)+\psi_{q^{2}}(k+1)\right) .
\end{aligned}
$$


Таким образом,

$$
\begin{aligned}
K_{n}^{(1)}\left(\left(1-q^{2}\right) z ; q^{2}\right)= & \frac{(-1)^{n} q^{-n^{2}+1 / 2}}{4 \pi a_{n}^{3}}\left(\left.\left(\frac{\partial}{\partial \nu} a_{\nu}-\frac{\partial}{\partial \nu} a_{-\nu}\right)\right|_{\nu=n} I_{n}^{(1)}\left(\left(1-q^{2}\right) z ; q^{2}\right)\right. \\
& \left.+\left.a_{n}\left(\frac{\partial}{\partial \nu} I_{-\nu}^{(1)}\left(\left(1-q^{2}\right) z ; q^{2}\right)-\frac{\partial}{\partial \nu} I_{\nu}^{(1)}\left(\left(1-q^{2}\right) z ; q^{2}\right)\right)\right|_{\nu=n}\right) .
\end{aligned}
$$

Из леммы 5.1 и (55) получим окончательное выражение

$$
\begin{aligned}
& K_{n}^{(1)}\left(\left(1-q^{2}\right) z ; q^{2}\right)=\frac{(-1)^{n} q^{-n^{2}+1 / 2}}{4 \pi a_{n}^{2}}\left(\left(\widetilde{a}-2 \ln \left(\frac{z}{2}\right)\right) I_{n}^{(1)}\left(\left(1-q^{2}\right) z ; q^{2}\right)\right. \\
& +\ln q^{2} \sum_{k=0}^{n-1}(-1)^{n-k-1} \frac{q^{-(n-k-1)(n-k-2)}\left(1-q^{2}\right)^{-n+2 k}\left(q^{2}, q^{2}\right)_{n-k-1}}{\left(q^{2}, q^{2}\right)_{k}}\left(\frac{z}{2}\right)^{-n+2 k} \\
& \left.+\sum_{k=0}^{\infty} \frac{\left(1-q^{2}\right)^{n+2 k}(z / 2)^{n+2 k}}{\left(q^{2}, q^{2}\right)_{k}\left(q^{2}, q^{2}\right)_{n+k}}\left(\psi_{q^{2}}(n+k+1)+\psi_{q^{2}}(k+1)\right)\right) .
\end{aligned}
$$

Для $j=2$ имеем

$$
\begin{aligned}
& K_{n}^{(2)}\left(\left(1-q^{2}\right) z ; q^{2}\right)=\frac{(-1)^{n} q^{-n^{2}+1 / 2}}{4 \pi a_{n}^{2}}\left(\left(\widetilde{a}-2 \ln \left(\frac{z}{2}\right)\right) I_{n}^{(2)}\left(\left(1-q^{2}\right) z ; q^{2}\right)\right. \\
& +\ln q^{2} \sum_{k=0}^{n-1}(-1)^{n-k-1} \frac{q^{-(n-k-1)(n-k-2)+2 k(k-n)}\left(1-q^{2}\right)^{-n+2 k}\left(q^{2}, q^{2}\right)_{n-k-1}}{\left(q^{2}, q^{2}\right)_{k}} \\
& \left.+\sum_{k=0}^{\infty} \frac{q^{2 k(n+k)}\left(1-q^{2}\right)^{n+2 k}(z / 2)^{n+2 k}}{\left(q^{2}, q^{2}\right)_{k}\left(q^{2}, q^{2}\right)_{n+k}}\left(\psi_{q^{2}}(n+k+1)+\psi_{q^{2}}(k+1)\right)\right) .
\end{aligned}
$$

ПРЕДЛОЖЕНИЕ 5.1. q-ФМ $K_{\nu}^{(1)}\left(\left(1-q^{2}\right) z ; q^{2}\right)$ является голоморфной функчией в области $\operatorname{Re} z>2 q /\left(1-q^{2}\right)$.

ДоказАТЕЛЬСтво. Подставим (38) в (54). Тогда

$$
K_{\nu}^{(1)}\left(\left(1-q^{2}\right) z ; q^{2}\right)=\frac{q^{-\nu^{2}+1 / 2}}{2 \sqrt{a_{\nu} a_{-\nu}} \sqrt{z}} e_{q}\left(-\frac{1-q^{2}}{2} z\right) \Phi_{\nu}(z)
$$

Произведение $e_{q}\left(-\frac{1-q^{2}}{2} z\right)$ и ${ }_{2} \Phi_{1}\left(q^{\nu+1 / 2}, q^{-\nu+1 / 2} ;-q ; q, \frac{2 q}{\left(1-q^{2}\right) z}\right)$ является голоморфной функцией в области $\operatorname{Re} z>2 q /\left(1-q^{2}\right)$.

ПРЕДЛОЖЕНИЕ 5.2. $q$-ФМ $K_{\nu}^{(2)}\left(\left(1-q^{2}\right) z ; q^{2}\right)$ является голоморфной функиией в области $z \neq 0$.

ДокАЗАТЕльСтво. Это утверждение следует из (54) и (18). 
СлЕДСТВИЕ 5.1.

$$
K_{\nu}^{(2)}\left(\left(1-q^{2}\right) z ; q^{2}\right)=\frac{q^{-\nu^{2}+1 / 2}}{2 \sqrt{a_{\nu} a_{-\nu}} \sqrt{z}} E_{q}\left(-\frac{1-q^{2}}{2} z\right) \Phi_{\nu}(z) .
$$

ДоКАЗАТЕЛЬство. Эта формула вытекает из (54) и (49).

ПРЕДЛОЖЕНИЕ 5.3. Функиия $K_{\nu}^{(1)}\left(\left(1-q^{2}\right) z ; q^{2}\right)$ удовлетворяет соотношения.м

$$
\begin{gathered}
\frac{2}{(1+q) z} \partial_{q} z^{\nu} K_{\nu}^{(1)}\left(\left(1-q^{2}\right) z ; q^{2}\right)=-z^{\nu-1} K_{\nu-1}\left(\left(1-q^{2}\right) z ; q^{2}\right) \\
\frac{2}{(1+q) z} \partial_{q} z^{-\nu} K_{\nu}^{(1)}\left(\left(1-q^{2}\right) z ; q^{2}\right)=-z^{-\nu-1} K_{\nu+1}\left(\left(1-q^{2}\right) z ; q^{2}\right) .
\end{gathered}
$$

ДоКАЗАТЕЛЬСТВО. Из определения 5.1 и предложения 3.1

$$
\begin{aligned}
& \frac{2}{(1+q) z} \partial_{q} z^{\nu} K_{\nu}^{(1)}\left(\left(1-q^{2}\right) z ; q^{2}\right) \\
& \quad=\frac{q^{-\nu^{2}+1 / 2}}{4\left(a_{\nu} a_{-\nu}\right)^{3 / 2} \sin \nu \pi}\left(a_{\nu} \frac{2}{(1+q) z} \partial_{q} z^{\nu} I_{-\nu}^{(1)}-a_{-\nu} \frac{2}{(1+q) z} \partial_{q} z^{\nu} I_{\nu}^{(1)}\right) \\
& \quad=\frac{q^{-\nu^{2}+1 / 2}}{4\left(a_{\nu} a_{-\nu}\right)^{3 / 2} \sin \nu \pi}\left(a_{\nu} z^{\nu-1} I_{-\nu+1}^{(1)}-a_{-\nu} z^{\nu-1} I_{\nu-1}^{(1)}\right) .
\end{aligned}
$$

Как следует из (44) $a_{\nu}=a_{\nu-1} q^{-\nu+1 / 2}, a_{-\nu}=a_{-\nu+1} q^{-\nu+1 / 2}$. Таким образом,

$$
\begin{aligned}
\frac{2}{(1+q) z} \partial_{q} z^{\nu} K_{\nu}^{(1)}= & -z^{\nu-1} \frac{q^{-(\nu-1)^{2}+1 / 2}}{4\left(a_{\nu-1} a_{-\nu+1}\right)^{3 / 2} \sin (\nu-1) \pi} \\
& \times\left(a_{\nu-1} z^{\nu-1} I_{-\nu+1}^{(1)}-a_{-\nu+1} z^{\nu-1} I_{\nu-1}^{(1)}\right) \\
= & -z^{\nu-1} K_{\nu-1}^{(1)} .
\end{aligned}
$$

Легко видеть, что $K_{-\nu}^{(j)}\left(\left(1-q^{2}\right) z ; q^{2}\right)=K_{\nu}^{(j)}\left(\left(1-q^{2}\right) z ; q^{2}\right)$. Тогда из (58)

$$
\frac{2}{(1+q) z} \partial_{q} z^{-\nu} K_{\nu}^{(1)}=\frac{2}{(1+q) z} \partial_{q} z^{-\nu} K_{-\nu}^{(1)}=-z^{-\nu-1} K_{-\nu-1}^{(1)}=-z^{-\nu-1} K_{\nu+1}^{(1)} .
$$

ПРЕДЛОЖЕНИЕ 5.4. Функция $K_{\nu}^{(1)}\left(\left(1-q^{2}\right) z ; q^{2}\right)$ удовлетворяет соотношениям

$$
\begin{aligned}
& K_{\nu-1}^{(1)}(\left.\left(1-q^{2}\right) z ; q^{2}\right)-K_{\nu+1}^{(1)}\left(\left(1-q^{2}\right) z ; q^{2}\right) \\
&=-\frac{2}{\left(1-q^{2}\right) z}\left(q^{-\nu}-q^{\nu}\right) K_{\nu}^{(1)}\left(\left(1-q^{2}\right) q z ; q^{2}\right), \\
& K_{\nu-1}^{(1)}\left(\left(1-q^{2}\right) z ; q^{2}\right)+K_{\nu+1}^{(1)}\left(\left(1-q^{2}\right) z ; q^{2}\right) \\
&=-\frac{4}{\left(1-q^{2}\right) z} K_{\nu}^{(1)}\left(\left(1-q^{2}\right) z ; q^{2}\right) \\
& \quad+\frac{2}{\left(1-q^{2}\right) z}\left(q^{-\nu}+q^{\nu}\right) K_{\nu}^{(1)}\left(\left(1-q^{2}\right) q z ; q^{2}\right) .
\end{aligned}
$$


ДокАЗАТЕЛЬСТво. Обозначим коэффициент перед квадратными скобками в (54) через $A_{\nu}$. Тогда из (44)

$$
A_{\nu-1}=-A_{\nu} q^{-\nu+1 / 2}, \quad A_{\nu+1}=-A_{\nu} q^{\nu+1 / 2} .
$$

Утверждение предложения вытекает из (44), (54), (21) и (22).

ПрЕДЛОЖЕНИЕ 5.5. Функция $K_{\nu}^{(2)}\left(\left(1-q^{2}\right) z ; q^{2}\right)$ удовлетворяет соотношениям

$$
\begin{gathered}
\frac{2}{(1+q) z} \partial_{q} z^{\nu} K_{\nu}^{(2)}\left(\left(1-q^{2}\right) z ; q^{2}\right)=-q^{-\nu+1} z^{\nu-1} K_{\nu-1}^{(2)}\left(\left(1-q^{2}\right) z ; q^{2}\right) \\
\frac{2}{(1+q) z} \partial_{q} z^{-\nu} K_{\nu}^{(2)}\left(\left(1-q^{2}\right) z ; q^{2}\right)=-q^{-\nu+1} z^{-\nu-1} K_{\nu+1}^{(2)}\left(\left(1-q^{2}\right) z ; q^{2}\right)
\end{gathered}
$$

ПРЕДЛОЖЕНИЕ 5.6. Функция $K_{\nu}^{(2)}\left(\left(1-q^{2}\right) z ; q^{2}\right)$ удовлетворяет соотношениям

$$
\begin{aligned}
& K_{\nu-1}^{(2)}(\left.\left(1-q^{2}\right) z ; q^{2}\right)-K_{\nu+1}^{(2)}\left(\left(1-q^{2}\right) z ; q^{2}\right) \\
&=-\frac{2 q^{\nu-1}}{\left(1-q^{2}\right) z}\left(q^{-\nu}-q^{\nu}\right) K_{\nu}^{(2)}\left(\left(1-q^{2}\right) q z ; q^{2}\right), \\
& K_{\nu-1}^{(2)}\left(\left(1-q^{2}\right) z ; q^{2}\right)+K_{\nu+1}^{(2)}\left(\left(1-q^{2}\right) z ; q^{2}\right) \\
&=-\frac{4 q^{\nu-1}}{\left(1-q^{2}\right) z} K_{\nu}^{(2)}\left(\left(1-q^{2}\right) z ; q^{2}\right) \\
& \quad+\frac{2 q^{\nu-1}}{\left(1-q^{2}\right) z}\left(q^{-\nu}+q^{\nu}\right) K_{\nu}^{(2)}\left(\left(1-q^{2}\right) q z ; q^{2}\right) .
\end{aligned}
$$

Предложения 5.5 и 5.6 доказываются так же, как и предложения 5.3 и 5.4 .

ПРЕДЛОЖЕНИЕ 5.7. Для любого $\nu$

$$
I_{\nu}^{(1)}\left(\left(1-q^{2}\right) z ; q^{2}\right) \quad \text { u } \quad K_{\nu}^{(1)}\left(\left(1-q^{2}\right) z ; q^{2}\right)
$$

образуют фундаментальную систему решений уравнения (23).

ДокАЗАтЕльство. Так как $K_{\nu}^{(1)}\left(\left(1-q^{2}\right) z ; q^{2}\right)$ является решением уравнения (23), $q$-вронскиан $W\left(I_{\nu}^{(1)}, K_{\nu}^{(1)}\right)(z)$ может быть записан в виде

$$
W\left(I_{\nu}^{(1)}, K_{\nu}^{(1)}\right)(z)=D_{\nu} e_{q^{2}}\left(\frac{\left(1-q^{2}\right)^{2}}{4} z^{2}\right)
$$

С другой стороны, из (38) и (56) следует, что

$$
\begin{aligned}
W\left(I_{\nu}^{(1)}, K_{\nu}^{(1)}\right)(z) & =\frac{q^{-\nu^{2}}}{2} \sqrt{\frac{a_{\nu}}{a_{-\nu}}} e_{q^{2}}\left(\frac{\left(1-q^{2}\right)^{2}}{4} z^{2}\right) \\
& =\left[\left(\frac{1}{2}+\frac{1-q^{2}}{2}\right) \Phi_{\nu}(z) \Phi_{\nu}(-q z)-\left(\frac{1}{2}-\frac{1-q^{2}}{2}\right) \Phi_{\nu}(-z) \Phi_{\nu}(q z)\right] .
\end{aligned}
$$

Следовательно, выражение в квадратных скобках есть константа. Пусть $z$ стремится к бесконечности. Тогда эта константа равна $1-q^{2}$ и

$$
W\left(I_{\nu}^{(1)}, K_{\nu}^{(1)}\right)(z)=\frac{q^{-\nu^{2}}\left(1-q^{2}\right)}{2} \sqrt{\frac{a_{\nu}}{a_{-\nu}}} e_{q^{2}}\left(\frac{\left(1-q^{2}\right)^{2}}{4} z^{2}\right) .
$$

Значит эта функция не обрашается в нуль. 
ПРЕДЛОЖЕНИЕ 5.8. Для любого $\nu$

$$
I_{\nu}^{(2)}\left(\left(1-q^{2}\right) z ; q^{2}\right) \quad u \quad K_{\nu}^{(2)}\left(\left(1-q^{2}\right) z ; q^{2}\right)
$$

образуют фундаментальную систему решений уравнения (27).

ДокАЗАТЕЛЬСТво. По аналогии с доказательством предложения 5.7 имеем

$$
W\left(I_{\nu}^{(2)}, K_{\nu}^{(2)}\right)(z)=\frac{q^{-\nu^{2}}\left(1-q^{2}\right)}{2} \sqrt{\frac{a_{\nu}}{a_{-\nu}}} E_{q^{2}}\left(-\frac{\left(1-q^{2}\right)^{2}}{4} z^{2}\right) .
$$

Эта функция не обрашается в нуль при $z \neq \pm 2 q^{-r} /\left(1-q^{2}\right), r=0,1, \ldots$.

ЗАмечАниЕ 5.1. Справедливо равенство

$$
\lim _{q \rightarrow 1-0} K_{\nu}^{(j)}\left(\left(1-q^{2}\right) z ; q^{2}\right)=K_{\nu}(z), \quad j=1,2 .
$$

Действительно, из (44) и (45) следует, что если $q=1$, то $a_{\nu}$ не зависит от $\nu$, и $a_{\nu}=1 / \sqrt{2 \pi}$. Теперь (59) следует из замечания 3.1 .

ЗАмЕчАнИЕ 5.2. Если $q \rightarrow 1-0$, представления (38) и (56) дают нам хорошо известные асимптотические разложения для функций $I_{\nu}(z)$ и $K_{\nu}(z)$, соответственHо $[13]$.

\section{§ 6. Некоторые соотношения для базисных гипергеометрических функций}

Дадим некоторые соотношения для базисных гипергеометрических функций, которые уже получены в $\S 4$. Заметим, что они основаны на отождествлении степенных рядов и лорановских разложений для одной и той же $q$-функции. Напомним, что

$$
\begin{gathered}
{ }_{2} \Phi_{1}\left(q^{\nu+1 / 2}, q^{-\nu+1 / 2} ;-q ; q, \frac{2 q}{\left(1-q^{2}\right) z}\right)=\Phi_{\nu}(z), \\
a_{\nu}=\sqrt{\frac{2}{1-q^{2}}} e_{q}(-1) \frac{I_{\nu}^{(2)}\left(2 ; q^{2}\right)}{\Phi_{\nu}\left(\frac{2}{1-q^{2}}\right)}, \quad \nu \neq n, \quad a_{n}=\sqrt{\frac{q^{-n^{2}+1 / 2} \ln q^{-2}}{2 \pi\left(1-q^{2}\right)}} .
\end{gathered}
$$

Положим в $(60) 2 q /\left(\left(1-q^{2}\right) z\right)=u,|u|<1$. Тогда из $(46)$ и (47)

$$
\begin{aligned}
& a_{\nu-1}{ }_{2} \Phi_{1}\left(q^{\nu-1 / 2}, q^{-\nu+3 / 2} ;-q ; q, u\right)-a_{\nu+12} \Phi_{1}\left(q^{\nu+3 / 2}, q^{-\nu-1 / 2} ;-q ; q, u\right) \\
& =a_{\nu} q^{-1 / 2}\left(q^{-\nu}-q^{\nu}\right)(u / q-1){ }_{2} \Phi_{1}\left(q^{\nu+1 / 2}, q^{-\nu+1 / 2} ;-q ; q, u / q\right), \\
& a_{\nu-12} \Phi_{1}\left(q^{\nu-1 / 2}, q^{-\nu+3 / 2} ;-q ; q, u\right)+a_{\nu+12} \Phi_{1}\left(q^{\nu+3 / 2}, q^{-\nu-1 / 2} ;-q ; q, u\right) \\
& =2 a_{\nu}(u / q)_{2} \Phi_{1}\left(q^{\nu+1 / 2}, q^{-\nu+1 / 2} ;-q ; q, u\right) \\
& \quad-a_{\nu} q^{-1 / 2}\left(q^{-\nu}+q^{\nu}\right)(u / q-1){ }_{2} \Phi_{1}\left(q^{\nu+1 / 2}, q^{-\nu+1 / 2} ;-q ; q, u / q\right) .
\end{aligned}
$$

Следуюшее соотношение, используюшее $q$-вронскиан (25), было получено для нецелых значений $\nu$

$$
\begin{aligned}
& (u / q+1)_{2} \Phi_{1}\left(q^{\nu+1 / 2}, q^{-\nu+1 / 2} ;-q ; q, u\right){ }_{2} \Phi_{1}\left(q^{\nu+1 / 2}, q^{-\nu+1 / 2} ;-q ; q,-u / q\right) \\
& -(u / q-1)_{2} \Phi_{1}\left(q^{\nu+1 / 2}, q^{-\nu+1 / 2} ;-q ; q,-u\right)_{2} \Phi_{1}\left(q^{\nu+1 / 2}, q^{-\nu+1 / 2} ;-q ; q, u / q\right)=2 .
\end{aligned}
$$

Так как $2 \Phi_{1}$ является непрерьвной функцией от $\nu$, это равенство остается справедливьп и для целых $\nu$. 


\section{Список литературы}

1. Jackson F. H. The application of basic numbers to Bessel's and Legendre's functions // Proc. London Math. Soc. (2). 1905. V. 2. P. 192-220.

2. Floreanini R., Vinet L. Addition formulas for $q$-Bessel functions // Preprint University of Montreal, UdeM-LPN-TH60, 1991; Representations of quantum algebras and $q$-special functions // Preprint Universite de Montreal, 1991.

3. Ваксман Л., Корогодский Л. Алгебра ограниченных функций на квантовой группе движений плоскости и $q$-аналоги функций Бесселя // ДАН СССР. 1989. Т. 304. № 5 . C. $1036-1040$.

4. Виленкин H. Я. Специальные функции и теория представлений групп. М.: Наука, 1990.

5. Дьнкин Е. Б. Неотрицательные собственные функции оператора Лапласа-Бельтрами на некоторых симметрических пространствах // ДАН СССР. 1961. Т. 141. № 2. C. 288-291.

6. Olshanetsky M. Martin boundaries for real semisimple Lie groups // J. Funct. Anal. 1994. V. 126. P. 169-216.

7. Jacquet H. P. B. Fonctions de Whittaker associees aux groupes de Chevalley // Bull. Soc. Math. France. 1967. V. 95. P. 243-309.

8. Schiffmann G. Integrales d'entrelacement et fonctions de Whittaker // Bull. Soc. Math. France. 1971. V. 99. P. 3-72.

9. Семенов-Тянь-Шанский $M$. Квантование открытой цепочки Тоды // Итоги науки и техники. Современные проблемы математики. Т. 16. М.: ВИНИТИ, 1987. С. 194-226.

10. Ginsparg P., Moore G. Lectures on 2d Gravity and 2d Strings // Preprint Yale Univ. TCTP-P23-92.

11. Olshanetsky M., Rogov $V$. Liouville quantum mechanics on a lattice from geometry of quantum Lorentz group // J. Phys. A. 1994. V. 27. P. 4669-4683.

12. Гаспер Дж., Рахман М. Базисные гипергеометрические ряды. М.: Мир, 1993.

13. Бейтмен Г., Эрдейи А. Высшие трансцендентные функции. Т. 2. М.: Наука, 1966.

Институт теоретической и экспериментальной физики;

Московский институт инженеров транспорта

E-mail: olshanez@vxdesy.desy.de; m10106@sucemi.bitnet
Поступила в редакцию 23.01 .1996 